\title{
Notificações da Hepatite B no Estado da Bahia, Brasil
}

\author{
Notification Hepatitis B in the state of Bahia, Brazil
}

Aline Pereira da Silva ${ }^{1}$, Bruna Almeida Argolo ${ }^{1}$, Caroline de Jesus Correia ${ }^{1}$, Flávia Tais Cantalice Sarmento $^{1}$, Janna Freire Andrade Lima ${ }^{1}$, Técia Maria Santos Carneiro e Cordeiro ${ }^{2 *}$, Argemiro D'Oliveira Júnior ${ }^{3}$

\author{
${ }^{1}$ Graduanda em Medicina. Faculdade de Medicina da Bahia. Universidade Federal da Bahia. ${ }^{2}$ Doutoranda \\ em Ciências da Saúde. Faculdade de Medicina da Bahia. Universidade Federal da Bahia. ${ }^{3}$ Doutor em \\ Medicina e Saúde. Professor Titular da Faculdade de Medicina da Bahia. Universidade Federal da Bahia.
}

\begin{abstract}
Resumo
Introdução: a Hepatite B é um problema de saúde pública no Brasil por ser prevenível. Objetivou-se analisar as associações entre as características individuais, sócio demográficas e os comportamentos de risco com os casos de Hepatite B no estado da Bahia. Metodologia: estudo epidemiológico de corte transversal exploratório. Todos os casos de VHB, notificados em 2014 no Sistema de Informação de Agravos de Notificação (SINAN), foram analisados com testes estatísticos para verificar as associações entre as variáveis. Resultados: a prevalência do $\mathrm{VHB}$, em relação às outras hepatites, foi de $32,6 \%$, atingindo pessoas, principalmente, com média de 37+13,92 anos de idade. Apresentou associação estatisticamente significante com a Hepatite B: o sexo masculino, a escolaridade com ensino médio completo, todas as faixas etárias, vacinação incompleta e não vacinados, fonte de infecção sexual e vertical, exposição a múltiplos parceiros e drogas injetáveis. Conclusão: a Hepatite B esteve associada a fatores individuais, sociodemográficos e comportamentos de risco entre a população estudada. Diante dos resultados percebe-se a necessidade de medidas de prevenção para o VHB, orientações acerca da doença e dos principais meios de infecção, diagnóstico precoce oportuno e a vacinação contra hepatite $B$ em vários ambientes comunitários. É preciso capacitação dos profissionais de saúde para notificação adequada e completa, assim como a vigilância desta infecção para facilitar o planejamento de medidas adequadas de controle e prevenção.
\end{abstract}

Palavras-chave: Vírus da Hepatite B. Notificação de Doenças. Transmissão. Comportamento de Risco.

\begin{abstract}
Introduction: Hepatitis B is a major public health problem in Brazil because it is preventable. This study aimed to examine the associations between individual characteristics, socio-demographic and the risk behavior with the cases of Hepatitis $B$ in the state of Bahia. Methodology: epidemiological study of exploratory cross-section. All cases of HBV reported in 2014 in the Notifiable Diseases Information System (SINAN) were analyzed with statistical tests to verify the associations between variables. Results: the prevalence of $\mathrm{HBV}$ in relation to other hepatitis, was 32.6\%, reaching people, mainly with mean $37+13.92$ years old. Showed a statistically significant association with hepatitis B: male, education with high school education, all age groups, incompletely vaccinated and unvaccinated, sexual and vertical infection source, exposure to multiple partners and injecting drugs. Conclusion: Hepatitis $B$ has been associated with individual factors, sociodemographic and risk behavior among the study population. Based on the results we see the need for prevention measures for $H B V$, guidelines about the disease and the main means of infection, appropriate early diagnosis and vaccination against hepatitis B in various community settings. It takes training of health professionals for proper and complete notification and the monitoring of this infection to facilitate the planning of appropriate measures for control and prevention.

Keywords: Hepatitis B Virus. Disease Notification. Transmission. Risk-Taking.
\end{abstract}

\section{INTRODUÇÃO}

As hepatites virais são doenças que apresentaram mudanças epidemiológicas nos últimos anos, sendo consideradas como um problema de saúde pública no Brasil. São doenças causadas por diversos agentes etiológicos, porém o hepatotropismo ocorre em todos os tipos. É uma doença de distribuição universal causada

Correspondente/Corresponding: *Técia Maria Santos Carneiro e Cordeiro - Programa de Pós-Graduação em Ciências da Saúde. Faculdade de Medicina da Bahia. End: Praça Conselheiro Almeida Couto, Largo do Terreiro de Jesus, s/n, Centro Histórico, Salvador-BA. CEP: 40025-010. Tel: 055 (75) 3283-5582 -E-mail: teciamarya@yahoo.com.br. pelos vírus $A, B, C, D$ e E, sendo a maior diferença entre elas a distribuição epidemiológica e a evolução clínica. A apresentação clínica pode ser desde a forma assintomática até as complicações como cirrose e carcinoma hepatocelular ${ }^{1,2}$.

Dentre os vírus causadores das hepatites virais, o Vírus da Hepatite B (VHB) é um vírus da família Hepadnaviridae formado por ácido desoxirribonucléico (DNA), sendo que após a infecção ocorre o tropismo no fígado e seu DNA contribuirá para os hepatócitos desenvolverem novos vírus ${ }^{3}$. A forma aguda ictérica da Hepatite B aumenta de acordo com a idade do indivíduo, já a forma crônica tem possibilidade de infecção quanto 
menor for a idade do paciente. Tem-se determinado grupos de risco para adquirir a Hepatite B de acordo os comportamentos das pessoas como os profissionais da área da saúde, homossexuais masculinos, usuários de drogas intravenosas, profissionais do sexo e pacientes em hemodiálise, entre outros ${ }^{1}$.

A transmissão desse vírus é por múltiplas formas, podendo ocorrer através do contato com a pele não íntegra, com mucosas, pela transmissão vertical, relações sexuais, exposição perfuro cutânea, tatuagem, piercings, transfusão de sangue e hemoderivados, dentre outros..$^{1,4}$ Estudo aponta que no Brasil, em 2011, a positividade para o VHB foi de $1,1 \%$ entre as pessoas de 10 a 19 anos e $11,6 \%$ para aqueles de 20 a 69 anos $^{4}$.

A Região Sul do Brasil foi referida como uma área de baixa endemicidade por apresentar prevalência de $\mathrm{HBsAg}$ reagente menor de $2 \%$ na população, enquanto as regiões Centro-Oeste, Nordeste e Sudeste como áreas de endemicidade intermediária pela prevalência de HBsAg reagente de $2-7 \%$. Localidades específicas no Brasil como a Amazônia Legal, o Estado do Espírito Santo e o Oeste do Estado de Santa Catarina foram considerados de alta endemicidade ao considerar a prevalência de HBsAg maior que $7 \%^{5}$.

Diante desta situação e ao considerar as Hepatites Virais um problema de Saúde Pública, este estudo se justifica pela necessidade de investigar as notificações da Hepatite B por se tratar de uma doença endêmica na Bahia e buscar relações entre a presença do vírus e aspectos predominantes nos casos. Desta forma, o presente estudo objetivou analisar as associações entre as características individuais, sóciodemográficas e os comportamentos de risco com os casos de Hepatite $B$ no estado da Bahia.

\section{METODOLOGIA}

Este é um estudo epidemiológico de corte transversal exploratório dos dados do Sistema de Informação de Agravos de Notificação (SINAN). Trata-se de um recorte do projeto de pesquisa "Aspectos epidemiológicos das notificações por Hepatites Virais no Brasil" desenvolvido no Programa de Pós-Graduação em Ciências da Saúde na Universidade Federal da Bahia.

Foram incluídos no estudo todos os casos de hepatites virais, notificados no SINAN no ano de 2014, na Bahia. O estado da Bahia é constituído de 417 municípios com uma população estimada para 2015 de 15.203.934 habitantes ${ }^{6}$.

Foram coletados os dados no banco de dados do SINAN fornecido pelo Ministério da Saúde através de autorização dos pesquisadores responsáveis. Por se tratar de um estudo exploratório foi definida a variável dependente do estudo e as variáveis exploratórias, as quais estão contidas na ficha de notificação de Hepatites Virais.

Desta forma, a variável dependente foi a Hepatite Viral do tipo B (Hepatite B e outros tipos de Hepatites Virais). As variáveis exploratórias foram: sexo (masculino e feminino), raça (negra, não negra e indígena), escolaridade (analfabeto, ensino fundamental incompleto, ensino fundamental completo, ensino médio completo e ensino superior completo), faixa etária (até 9 anos, 10 a 19 anos, 20 a 59 anos, 60 anos e mais), vacina da Hepatite $B$ (completa, incompleta e não vacinado), provável fonte de infecção (sexual, transfusional, vertical, acidentes de trabalho, hemodiálise, domiciliar, tratamento cirúrgico, tratamento dentário, pessoa/pessoa, alimento/água contaminada, e outros), exposto a três ou mais parceiros sexuais (sim e não), agravos associados a HIV/AIDS (sim e não), exposto a drogas injetáveis (sim e não) e exposto a tatuagem e piercing (sim e não).

Os dados deste estudo foram processados pelos programas Microsoft Office Excel versão 2007 e o OpenEpi versão 3.03a. A análise dos dados deu-se pela análise descritiva univariada com as estimativas das frequências absolutas, relativas, moda, média e desvio padrão e a análise bivariada pelo teste Qui-quadrado considerando a significância estatística valores $>3,841$ e o valor de $\mathrm{P}<0,05$ na direção bicaudal, quando os valores esperados foram $<5$ utilizou-se exato de Fisher.

A Resolução 466/2012 foi respeitada de acordo os aspectos éticos em pesquisas com seres humanos, sendo que este estudo foi aprovado pelo Comitê de Ética em Pesquisa (CEP) da Faculdade de Medicina da Bahia / Universidade Federal da Bahia sob protocolo de no 1.249.977/2015.

\section{RESULTADOS}

O estudo foi realizado com 1.699 indivíduos notificados com hepatites virais em 2014, dos quais 553 tinham Hepatite B. Dessa forma, a prevalência da Hepatite B em comparação às outras hepatites, foi de $32,6 \%$. Com relação à idade do diagnóstico da Hepatite $\mathrm{B}$, observou-se que o mesmo ocorreu em pessoas com média de 37 $\pm 13,92$ anos, sendo mais frequente com 29 anos.

Quanto às variáveis sociodemográficas, houve associação estatisticamente significante entre a Hepatite $B$ e o sexo masculino $(P=0,003)$, nível de escolaridade ensino médio completo $(P=0,009)$ e em todas as faixas etárias, sendo de maior proporção na faixa de 20 a 59 anos $(\mathrm{P}<0,001)$ (Tabela 1$)$. 
Tabela 1 - Características sociodemográficas e a Hepatite B, Bahia, 2014.

\begin{tabular}{|c|c|c|c|c|c|}
\hline \multirow{2}{*}{ Variáveis } & Hepatite B & Outros tipos de hepatites & \multirow{2}{*}{ Total } & \multirow{2}{*}{ Qui-quadrado } & \multirow{2}{*}{ Valor de $\mathrm{P}$} \\
\hline & $\mathrm{n}(\%)$ & $\mathrm{n}(\%)$ & & & \\
\hline \multicolumn{6}{|l|}{ Sexo } \\
\hline Feminino & $290(38,5 \%)$ & $463(61,5 \%)$ & 753 & - & - \\
\hline Masculino & $263(31,4 \%)$ & $575(68,6 \%)$ & 838 & 8,887 & 0,003 \\
\hline \multicolumn{6}{|l|}{ Raça } \\
\hline Negra & $427(34,4 \%)$ & $813(65,6 \%)$ & 1240 & 0,781 & 0,377 \\
\hline Não negra & $49(26,6 \%)$ & $135(73,4 \%)$ & 184 & 1,432 & 0,236 \\
\hline Indígena & $6(46,2 \%)$ & $7(53,8 \%)$ & 13 & - & - \\
\hline \multicolumn{6}{|l|}{ Escolaridade } \\
\hline Analfabeto & $14(45,2 \%)$ & $17(54,8 \%)$ & 31 & 3,378 & 0,066 \\
\hline Ensino fundamental incompleto & $172(34,6 \%)$ & $325(65,4 \%)$ & 497 & 1,719 & 0,190 \\
\hline Ensino fundamental completo & $51(38,3 \%)$ & $82(61,7 \%)$ & 133 & 2,686 & 0,101 \\
\hline Ensino médio completo & $108(45,2 \%)$ & $131(54,8 \%)$ & 239 & 6,707 & 0,009 \\
\hline Ensino superior completo & $13(25,5 \%)$ & $38(75,5 \%)$ & 51 & - & - \\
\hline \multicolumn{6}{|l|}{ Faixa etária } \\
\hline Até 9 anos & $4(1,4 \%)$ & $292(98,6 \%)$ & 296 & - & - \\
\hline 10 a 19 anos & $38(22,5 \%)$ & $131(77,5 \%)$ & 169 & 58,48 & $<0,001$ \\
\hline 20 a 59 anos & $470(49,1 \%)$ & $487(50,8 \%)$ & 957 & 219,3 & $<0,001$ \\
\hline 60 anos e mais & $41(24,3 \%)$ & $128(75,7 \%)$ & 169 & 64,59 & $<0,001$ \\
\hline
\end{tabular}

Fonte: Dados da pesquisa

Em relação aos comportamentos de risco, a vacinação incompleta $(P<0,001)$ e não vacinados $(P<0,001)$ contra a VHB mostrou-se associada a Hepatite B. As prováveis fontes de infecção, apresentaram-se associadas à Hepatite $B$ a infecção por via sexual $(P=0,006)$ e vertical
$(P=0,021)$. A exposição a três ou mais parceiros sexuais esteve associada com o alto risco de contaminação com o VHB $(P=0,001)$. A exposição a drogas injetáveis, também, esteve associada à contaminação com o VHB $(P=0,001)$ em relação às outras hepatites (Tabela 2 ).

Tabela 2 - Comportamentos de risco e a Hepatite B, Bahia, 2014.

\begin{tabular}{|c|c|c|c|c|c|}
\hline \multirow{2}{*}{ Variáveis } & Hepatite B & Outros tipos de hepatites & \multirow{2}{*}{ Total } & \multirow{2}{*}{ Qui-quadrado } & \multirow{2}{*}{ Valor de $\mathrm{P}$} \\
\hline & n (\%) & $\mathrm{n}(\%)$ & & & \\
\hline \multicolumn{6}{|l|}{ Vacina Hepatite B } \\
\hline Completa & $67(13,3 \%)$ & $437(86,7 \%)$ & 504 & - & - \\
\hline Incompleta & $101(50,8 \%)$ & $98(49,2 \%)$ & 199 & 110,1 & $<0,001$ \\
\hline Não vacinado & $170(48,7 \%)$ & $179(51,3 \%)$ & 349 & 128,9 & $<0,001$ \\
\hline \multicolumn{6}{|l|}{ Provável fonte de infecção } \\
\hline Sexual & $183(77,9 \%)$ & $52(22,1 \%)$ & 235 & 8,985 & 0,006 \\
\hline Transfusional & $13(16,3 \%)$ & $67(83,7 \%)$ & 80 & 0,298 & 0,999 \\
\hline Vertical & $8(88,9 \%)$ & $1(11,1 \%)$ & 9 & 5,104 & 0,021 \\
\hline Acidentes de trabalho & $6(60,0 \%)$ & $4(40,0 \%)$ & 10 & 1,371 & 0,241 \\
\hline Hemodiálise & $1(16,7 \%)$ & $5(83,3 \%)$ & 6 & - & - \\
\hline Domiciliar & $7(24,1 \%)$ & $22(75,9 \%)$ & 29 & 0,019 & 0,999 \\
\hline Tratamento cirúrgico & $8(38,1 \%)$ & $13(61,9 \%)$ & 21 & 0,241 & 0,646 \\
\hline Tratamento dentário & $23(53,5 \%)$ & $20(46,5 \%)$ & 43 & 1,573 & 0,208 \\
\hline Pessoa/pessoa & $5(19,2 \%)$ & $21(80,8 \%)$ & 26 & 0,189 & 0,999 \\
\hline Alimento/água contaminada & $1(0,3 \%)$ & $355(99,7 \%)$ & 356 & - & 0,065 \\
\hline Outros & $56(40,6 \%)$ & $82(59,4 \%)$ & 138 & 0,557 & 0,468 \\
\hline \multicolumn{6}{|c|}{ Exp. Três ou mais parceiros sexuais } \\
\hline Sim & $114(45,4 \%)$ & $137(54,6 \%)$ & 251 & 15,81 & 0,001 \\
\hline \multicolumn{6}{|l|}{ Agravos associados a HIV/AIDS } \\
\hline $\operatorname{Sim}$ & $15(40,5 \%)$ & $22(59,5 \%)$ & 37 & 0,523 & 0,469 \\
\hline \multicolumn{6}{|l|}{ Exp. Drogas injetáveis } \\
\hline Sim & $10(10,9 \%)$ & $82(89,1 \%)$ & 92 & 24,31 & 0,001 \\
\hline \multicolumn{6}{|l|}{ Exp. Tatuagem e piercing } \\
\hline $\operatorname{Sim}$ & $70(40,9 \%)$ & $101(59,1 \%)$ & 171 & 3,635 & 0,057 \\
\hline
\end{tabular}

Exp. = Exposição.

Fonte: Dados da pesquisa 


\section{DISCUSSÃO}

A Hepatite Viral B notificada no SINAN no estado da Bahia em 2014 esteve associada ao sexo masculino, a escolaridade, a faixa etária, a vacinação incompleta ou não vacinados, as fontes de infecção sexual e vertical e a exposição a múltiplos parceiros sexuais e a drogas injetáveis.

Foi observado que no Brasil, no ano de 2010 os casos de Hepatite $B$ foram mais numerosos no sexo feminino, sendo estes na faixa etária de 15 a 29 anos. ${ }^{7}$ Já o Boletim Epidemiológico de Hepatites Virais ${ }^{8}$ apresenta uma modificação deste perfil com a frequência da Hepatite $B$ maior em homens no Brasil o que corrobora com os resultados do presente estudo.A faixa etária entre 20 e 59 anos, que são os adultos, está mais vulnerável ao VHB. Outro estudo ${ }^{3}$ identificou a maior incidência de infectados na faixa etária entre 40 e 59 anos.

Os achados do presente estudo mostraram que o ensino médio completo esteve associado ao VHB, embora a menor prevalência da Hepatite $B$ foi entre pessoas com o ensino superior completo. Tal constatação é ratificada por outro estudo ${ }^{4}$, o qual afirma que indivíduos que possuem menor escolaridade a chance de risco de adquirir VHB é maior que os indivíduos que possuem maior escolaridade. A desigualdade pode favorecer no nível de conhecimento e prevenção.

A vacinação incompleta e não vacinados estiveram entre aqueles que apresentaram a maior frequência de Hepatite B, embora alguns casos mesmo vacinados desenvolveram a doença. A vacinação é uma medida de proteção específica na prevenção primária contra a infecção pelo VHB e, em consequência, para as complicações como o câncer. Porém, é recomendada a aplicação das três doses da vacina contra a Hepatite $B$, ou seja, o esquema completo e, posteriormente, deve-se realizar o teste sorológico Anti-HBs para verificar se o individuo está imunizado e com proteção garantida ${ }^{3,7,9}$. O calendário vacinal atual do Programa Nacional de Imunização ofertado pelo Sistema Único de Saúde incluiu todas as faixas etárias como população-alvo para vacinação contra Hepatite B, de preferência completando o esquema aos 6 meses de idade.

A vacina contra Hepatite $B$ em três ou quatro doses induz uma resposta satisfatória, com produção de anticorpos igual ou acima de $10 \mathrm{mUl} / \mathrm{ml}$, em mais de $90 \%$ dos adultos e $95 \%$ das crianças e adolescentes saudáveis. ${ }^{9} \mathrm{~A}$ imunogenicidade da vacina pode diminuir com o avançar da idade, com alguns hábitos de vida, como o tabagismo e etilismo, assim como em obesos, em portadores de doenças imunossupressoras, diabetes mellitus e insuficiência renal crônica. Além disso, uma parcela da população, que varia de 2,5 a $5 \%$, não responde à vacina contra Hepatite B, não adquirindo imunidade contra a doença. No Brasil, no período de $2014,95,82 \%$ da população brasileira foi vacinada contra a Hepatite B. Na região Nordeste, a cobertura vacinal foi de $94,20 \%$ e na Bahia de $92,41 \%^{10}$.
A vacinação é considerada eficiente na redução da morbidade e mortalidade das doenças infecciosas e um dos desafios é a adaptação do calendário vacinal ao contexto epidemiológico local ${ }^{11}$, o que tem sido alcançado no Brasil em relação a vacina contra Hepatite $B$.

As fontes de infecção sexual por múltiplos parceiros e vertical foram aquelas que estiveram associadas à Hepatite B. Embora estudos ${ }^{12,13}$ tenham demonstrado o grande risco de infecção pelo VHB em pessoas que estão em constante contato com sangue e seus derivados, como os trabalhadores da saúde, principalmente na área hospitalar. Percebe-se com os resultados do presente estudo que os baianos estão desenvolvendo comportamento de risco que os colocam em exposição ao VHB, assim como dos recém-nascidos.

A relação sexual é um meio importante de transmissão da Hepatite $B$, no presente estudo houve associação desta fonte de infecção, assim como exposição a múltiplos parceiros sexuais. Em Montes Claros de 2007 a 2015,14 $19,8 \%$ dos casos notificados com Hepatite B estavam expostos a três ou mais parceiros sexuais. Considera-se a via sexual uma forma de fácil prevenção pelo uso correto do preservativo feminino ou masculino. Ferreira e Silveira ${ }^{1}$ apresentam que tanto o sangue quanto os outros líquidos orgânicos das pessoas infectadas pelo VHB já podem ser infectantes mesmo antes de aparecer os sinais da doença, dessa forma se faz importante o uso de preservativos. No entanto, outros autores ${ }^{15}$ relatam que fatores socioeconômicos, demográficos, ambientais e hábitos de vida podem influenciar negativamente no conhecimento e uso do preservativo masculino em adolescentes. Esses fatores podem, então, estar associados também a uma maior transmissibilidade do VHB por via sexual. O uso irregular de preservativo também foi um fator associado à infecção por VHB em usuários de drogas ${ }^{16}$. Desse modo, os comportamentos de risco podem contribuir para infecção pelo VHB.

A transmissão vertical é uma forma também prevenível de transmissão do VHB. Considera-se transmissão vertical do VHB a que ocorre durante a gestação, parto, cuidados com o recém-nascido e aleitamento materno. A via de transmissão mais comum para ocorrência é no momento do parto ${ }^{15}$. Em Salvador de 2007 a $2012^{17}$ a transmissão vertical foi a fonte de infecção com maior proporção entre as mulheres $(62,5 \%)$ e houve quantitativo relevante de casos de Hepatite B em gestantes, com maior notificação no terceiro trimestre. Sendo este período o de maior risco de transmissão vertical pela replicação viral do antígeno causador da Hepatite B. Por isso é recomendado a sorologia para Hepatite $B$ durante o pré-natal, assim como a primeira dose da vacina contra a doença nas primeiras 12 horas de vida do recém-nascido.

O uso de drogas injetáveis é uma fonte de infecção que esteve associada à Hepatite $B$ no presente estudo. Em São Paulo ${ }^{18} 27,3 \%$ dos usuários de drogas injetáveis vivendo com HIV/Aids apresentaram infecção pela Hepatite B, com maior proporção entre os homens $(92,9 \%)$ 
do que entre as mulheres (7,1\%). Desde 1993 quando foi implantado o teste sorológico visando o controle das hepatites por transfusão sanguínea, observou-se que os usuários de drogas injetáveis era a população com maior percentual de novos casos. Este fato pode estar associado ao compartilhamento de frasco de droga e agulhas, além da falta de conhecimento acerca das hepatites, da limpeza das agulhas e seringas, entre outros. Achado diferente foi observado em Montes Claros $^{14}$ e em Salvador ${ }^{17}$ em que apenas $2,9 \%$ e $2,4 \%$ dos casos notificados estavam expostos a drogas injetáveis, respectivamente.

O diagnóstico precoce da Hepatite B é primordial ao considerar que portadores do VHB na infecção crônica, mesmo que não apresentem doença hepática ativa, possuem riscos para desenvolver complicações como a cirrose, a insuficiência hepática e o carcinoma hepatocelular ${ }^{19}$. Uma medida que apresenta eficácia e efetividade para a prevenção da Hepatite B é a vacinação contra o VHB para toda a população, principalmente para as pessoas que estão nos grupos de risco. Além disso, é necessária a prevenção para a infecção perinatal por meio da triagem dos marcadores sorológicos na gestante durante o pré-natal e, também, pelos testes pós-vacinação em recém-nascidos de mães com o vírus para avaliar a resposta dos lactentes a vacinação e a necessidade de revacinação ${ }^{1}$.

Uma forma de diagnóstico precoce implementada pelo Ministério da Saúde, recentemente, foram os testes rápidos para Hepatites $\mathrm{B}$ e $\mathrm{C}$. São testes rápidos, práticos e de fácil execução, pois não precisam de equipamentos para leitura. Os resultados são identificados pela formação de linhas coloridas no cartão de leitura visual de fácil interpretação. Esses testes rápidos utilizam a tecnologia imunocromatográfica, a qual possibilita a deteç̧ão de antígeno do HBs no soro, plasma ou sangue total. Contudo, os testes sorológicos específicos como o $\mathrm{HBsAg}$, Anti-HBc IgM e Anti-HBc total são mais eficazes para analisar a evolução da infecção viral e os procedimentos a serem seguidos ${ }^{20}$.

O presente estudo teve como limitação a impossibilidade de estabelecer relação temporal entre exposição ao fator de risco e o desenvolvimento da doença por se tratar de um corte transversal. Além disso, analisa casos de Hepatite B informados ao SINAN em 2014, dentre os quais podem haver subnotificações e notificações incompletas, situações que comprometem a qualidade do banco de dados. A comparação entre grupo de casos de Hepatite B com outros vírus de hepatites pode ser uma limitação da análise dos dados. No entanto, apresenta dados relevantes para a vigilância em saúde nos municípios baianos e de todo o mundo ao identificar os principais grupos vulneráveis a Hepatite $B$ e, em consequência, subsidiar as medidas de intervenção.

\section{CONCLUSÃO}

Conclui-se que a hepatite $B$ esteve associada a fatores individuais, sociodemográficos e comportamentos de risco entre a população estudada. Estes dados podem contribuir para intervenções na clínica e nas comunidades a fim de prevenir esta doença transmissível.

O diagnóstico precoce por meio dos testes rápidos e sorológicos deve ser realizado em momentos oportunos, assim como a vacinação em massa da população e os testes sorológicos para conferir imunidade. É necessária a educação em saúde considerando os aspectos sociais, culturais, econômicos, psicológicos e biológicos da população para favorecer nas orientações e sensibilização das formas de prevenção e controle desta doença infecciosa, a Hepatite B.

Adiciona-se também a capacitação dos profissionais de saúde para o diagnóstico oportuno, formação de educadores em saúde e o preenchimento adequado e completo das fichas de notificação para favorecer na atuação eficiente da vigilância desta doença infecciosa.

\section{REFERÊNCIAS}

1. FERREIRA, C. T.; SILVEIRA, T. R. Hepatites virais: aspectos da epidemiologia e da prevenção. Rev. Bras. Epidemiol., São Paulo, v. 7, n. 4, p. 473-487, 2004.

2. TAUIL, M. C. et al. Mortalidade por hepatite viral B no Brasil, 20002009. Cad. Saúde Pública, Rio de Janeiro, v. 28, n. 3, p. 172-178, 2012.

3. SILVA, A.C.L.G.; TOZATTI, F.; WELTER, A.C. Incidência e mortalidade por hepatite B, de 2001 a 2009: uma comparação entre Brasil, Santa Catarina e Florianópolis. Cad. Saúde Coletiva, Goiania, v. 21, n. 1, p. 34-39, 2013.

4. DIAS, J. A.; CERUTTI JÚNIOR, C.; FALQUETO, A. Fatores associados à infecção pelo vírus da hepatite $B$ : um estudo caso-controle no município de São Mateus, Espírito Santo. Epidemiol. Serv. Saúde, Brasília, v. 23, n. 4, p. 683-690, 2014.

5. CHÁVEZ, J. H.; CAMPANA, S. G.; HAAS, P. Panorama da Hepatite B no Brasil e no Estado de Santa Catarina. Rev. panam. salud. pública, Washington, v. 14, n. 2, p. 91-96, 2003.

6. INSTITUTO BRASILEIRO DE GEOGRAFIA E ESTATÍSTICA (IBGE). Estados@ - Bahia. Estimativas 2015. Disponível em: <http://www.ibge. gov.br/estadosat/perfil.php?lang=\&sigla=ba>. Acesso em: 20 abr. 2016.

7. ESPÍNDOLA, M. F. S.; MESENBURG, M.A.; SILVEIRA, M.F. Acesso à vacina contra a hepatite $B$ entre parturientes que realizaram o pré-natal em Pelotas, Rio Grande do Sul. Epidemiol. Serv. Saúde, Brasília, v. 23, n. 3, p. 447-454, 2014.

8. BRASIL. Ministério da Saúde. Hepatites Virais. Boletim Epidemiológico, v. IV, n. 1, p. 1-25, 2015.

9. MORAES, J.C.; LUNA, E.J.A.; GRIMALDI, R.A. Imunogenicidade da vacina brasileira contra hepatite $B$ em adultos. Rev. Saúde Pública, São Paulo, v. 44, n. 2, 353-359, 2010.

10. DEPARTAMENTO DE INFORMÁTICA DO SISTEMA ÚNICO DE SAÚDE. Informações de Saúde. Assistência à Saúde. Imunizações.Cobertura. Brasil. 2016. Disponível em: <http://www2.datasus.gov.br/DATASUS/ index.php?area $=0202 \& i d=11638 \& V O b j=h t t p: / /$ tabnet.datasus.gov.br/ cgi/deftohtm.exe?pni/cnv/cpni>. Acesso em: 12 ago. 2016.

11. LERNOUT, T. et al. Do vaccines save lives? yes they do! Acta Med. Port., Lisboa, v. 27, n. 2, p. 160-162, 2014.

12. FERNANDES, J. V. et al. Prevalência de marcadores sorológicos do vírus da hepatite $B$ em trabalhadores do serviço hospitalar. Rev. Saúde 
Pública, São Paulo, v. 33, n. 2, p. 122-128, 1999.

13. SILVA, F. J. C. P. et al. Estado vacinal e conhecimento dos profissionais de saúde sobre hepatite $B$ em um hospital público do nordeste brasileiro. Rev. bras. saúde ocup., São Paulo, v. 36, n. 124, p. 258-264, 2011.

14. PEREIRA, F. S. et al. Estilo de vida e exposição a material biológico entre notificados com hepatite B. J. Health Biol. Sci.,[s.I], v. 4, n. 2, p. 117-122, 2016.

15. HARTMANN, J. M.; CESAR, J. Q. Conhecimento de preservativo masculino entre adolescentes: estudo de base populacional no semiárido nordestino, Brasil. Cad. Saúde Pública, Rio de Janeiro, v. 29, n. 11, p. 2297-2306, 2013.

16. FERREIRA, R.C. et al. Prevalence of hepatitis $B$ virus and risk factors in Brazilian non-injecting drug users. J. Med. Virol., New York, v. 81, n. 4, p. 602-609, 2009.
17. MARTINS, M. M. F.; COSTA, E.A.M. Aspectos epidemiológicos e estado vacinal para Hepatite B no município de Salvador, Bahia. Rev. Ciênc. Méd. Biol., Santiago, v. 14, n. 2, p. 160-164, 2015.

18. MARCHESINI, A. M. et al. Hepatites B e C em usuários de drogas injetáveis vivendo com HIV em São Paulo, Brasil. Rev. Saúde Pública, São Paulo, v. 41, suppl. 2, p. 57-63, 2007.

19. FERREIRA, M.S. Diagnóstico e tratamento da hepatite B. Rev. Soc. Bras. Med. Trop., Brasília, v. 33, n. 4, p. 389-400, 2000.

20. BRASIL.Ministário da Saúde. Manual de treinamento para teste rápido hepatites B (HBsAg) e C (anti-HCV). Brasília: Ministério da Saúde, 2011. Disponível em: <http://www.aids.gov.br/sites/default/files/ anexos/page/2012/50770/manual_para_capacitacao_de_tr_para_as_ hepatites_b_17745.pdf>. Acesso em: 16 ago. 2016.

Submetido em: $15 / 11 / 2017$

Aceito em: 11/01/2018 